\title{
2x2 Array Multiplier Based on DCVS Logic
}

\author{
Ila Gupta \\ Deptt. Of ECE \\ FET-MITS (Deemed \\ University) \\ Lakshmangarh
}

\author{
Neha Arora \\ Deptt. Of ECE \\ FET-MITS (Deemed \\ University) \\ Lakshmangarh
}

\author{
Prof. B. P. Singh \\ Deptt. Of ECE \\ FET-MITS (Deemed University) \\ Lakshmangarh
}

\begin{abstract}
The Array architecture is a popular technique to implement the multipliers due to its compact structure. In this paper, 2x2 array multiplier circuits using existing full adder and DCVS logic full adder have been designed, simulated, analyzed and compared. An extensive analysis of multipliers has been done. According to our test results, an array multiplier designed by DCVS logic full adder is showing best result in terms of power consumption with varying supply voltage, temperature and operating frequency. The simulation has been carried out on Tanner EDA tool on BSIM3v3 90nm technology.
\end{abstract}

\section{Keywords: Array multiplier, Full adder cell, High speed, 2:1 Multiplexer, VLSI}

\section{INTRODUCTION}

Multiplication is one of the basic arithmetic operations. In fact $8.72 \%$ of all instructions in a typical scientific program are multiplies. It is the most important operation in digital computer systems and digital signal processors. Digital multipliers are the most commonly used components in many digital circuit designs[1]. They are fast, reliable and efficient components that are utilized to implement any operation. The basic building block of the multiplier is the full adder cell, thus it has a significant effect on the overall performance and speed of the multiplier. Therefore, high speed full adders based on DCVS logic were presented .This one bit full adder is based on DCVSL 2:1 multiplexer.

\section{DIFFERENTIAL CASCODE VOLTAGE} SWITCH LOGIC (DCVSL)

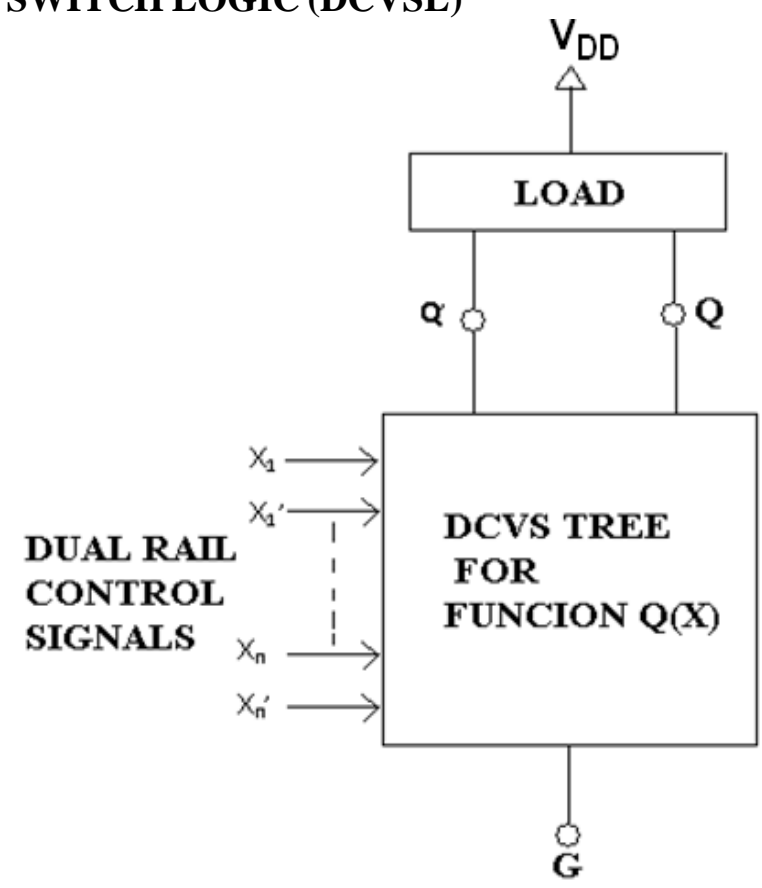

Fig.1 Basic DCVSL Circuit
One of the first realization of static differential CMOS logic known as the Differential Cascode Voltage Switch Logic (DCVSL) was introduced in 1984 [9]. Since then researchers have shown great interest in differential logic. This is due to its potential to efficiently realize complex logic functions. A logic function and its inverse are automatically implemented in this logic style. The pulldown networks implemented by the NMOS logic tree generated complementary output. The advantage of DCVSL is in its logic density that is achieved by elimination of large PFETS from each logic function. The basic DCVS circuit comprises two parts: a binary decision tree and a load. The tree is specified such that:

(1)When the input vector $X=(X 1, \ldots X n)$ is the true vector of the switching function $\mathrm{Q}(\mathrm{x})$, then the output $\mathrm{Q}$ is disconnected from node $\mathrm{G}$ and the node $\mathrm{Q}$ ' is connected to $\mathrm{G}$; and

(2) When $X=(X 1 \ldots X n)$ is the false vector of $Q(x)$, and then the reverse holds. [2]- [4] Fig.1 shows the basic DCVSL Circuit.

The paper is organized as follows. First, Section II introduces the basics of Differential Cascode Voltage Switch Logic, Section III introduces the existing 2X2 array multiplier design then Section IV presents the designed 2x2 array multipliers, Section $\mathrm{V}$ shows simulations results and comparison and then finally conclusion is written in Section V.

\section{EXISTING 2X2 ARRAY MULTIPLIER DESIGN}

The basic process of binary array multiplication involves the AND operation of multiplicand and multiplier bits and subsequent addition. $\mathrm{A}(\mathrm{A} 1, \mathrm{~A} 0)$ and $\mathrm{B}(\mathrm{B} 1, \mathrm{~B} 0)$ are 2-bit inputs given to multiplier giving output $\mathrm{M}$ (M3, M2, M1, M0). Fig. 2 shows the basic architecture representing $2 \times 2$ array multiplier. It consists of four 2-input AND gates and 
two 1-bit full adders. Multiplier design can be divided into two blocks, product generation and product addition. Product generation part built with the help of And Gate and product addition part built with the help of one bit full adder cell. Fig. 3 shows the block diagram of existing $2 \times 2$ array multiplexer. This diagram contains 8 instances of CMOS inverter, 4 instances of NOR Gate and 2 instances of existing 1 bit full adder cell. The AND Gate formed with the help of CMOS. [5]

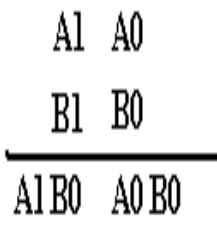

$\mathrm{AlBl} A 0 \mathrm{Bl}$

M3 M2 Ml M0

Fig. 2 X2 multiplication

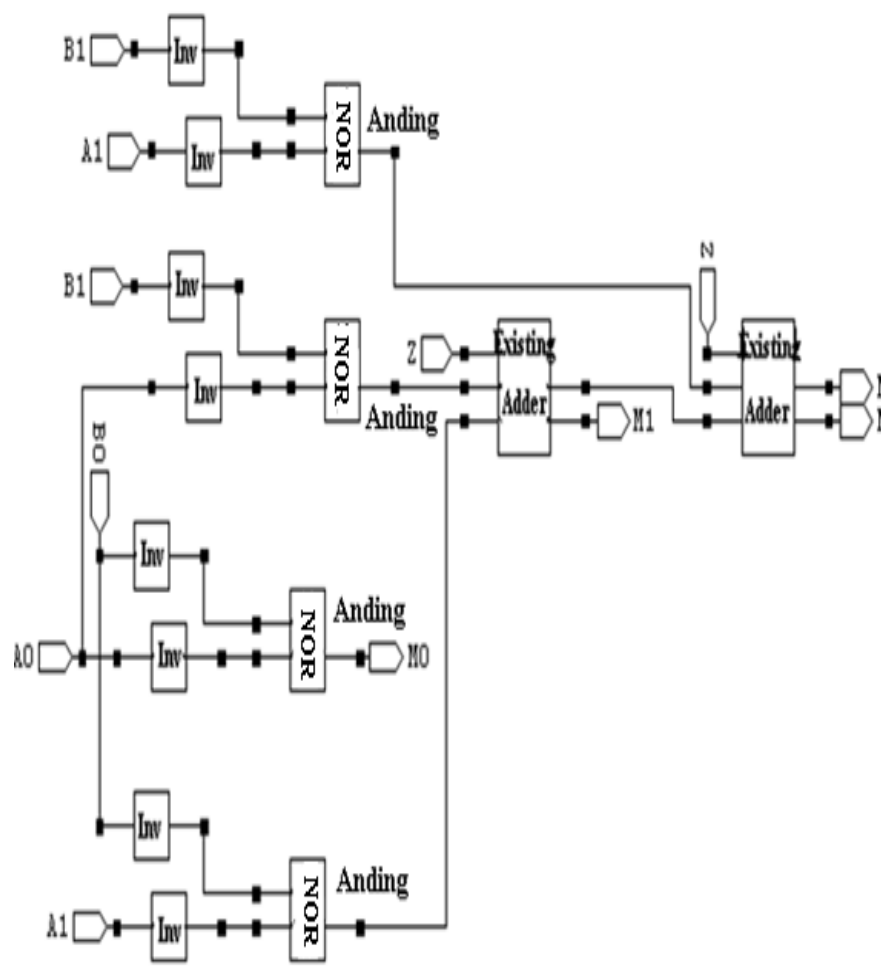

Fig.3 Schematic of existing adder circuit

inverter and NOR Gate. The schematic diagram and instances of inverter, NOR Gate and existing one bit full adder cell is shown in Fig.4, Fig.5, Fig.6, Fig7, Fig.8 and Fig. 9 respectively.

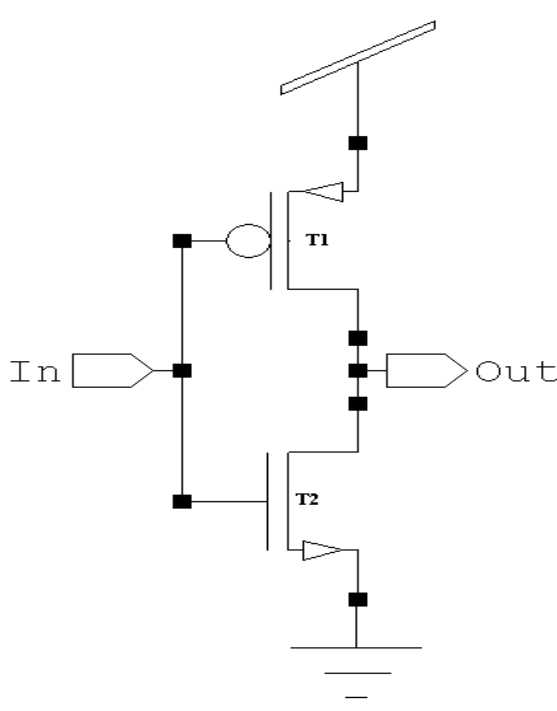

Fig.4 Schematic of CMOS inverter

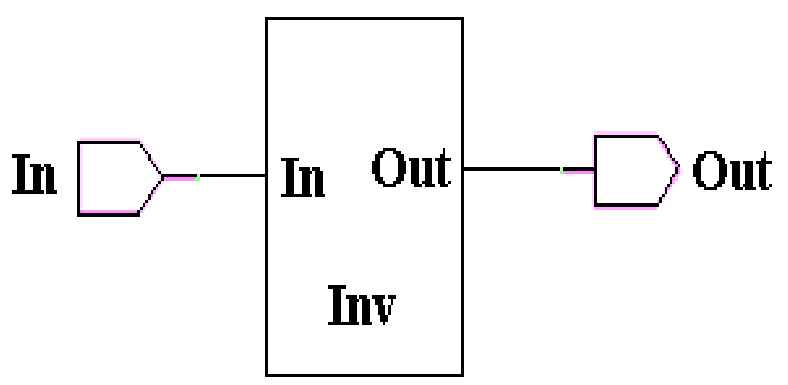

Fig.5 Instance of inverter

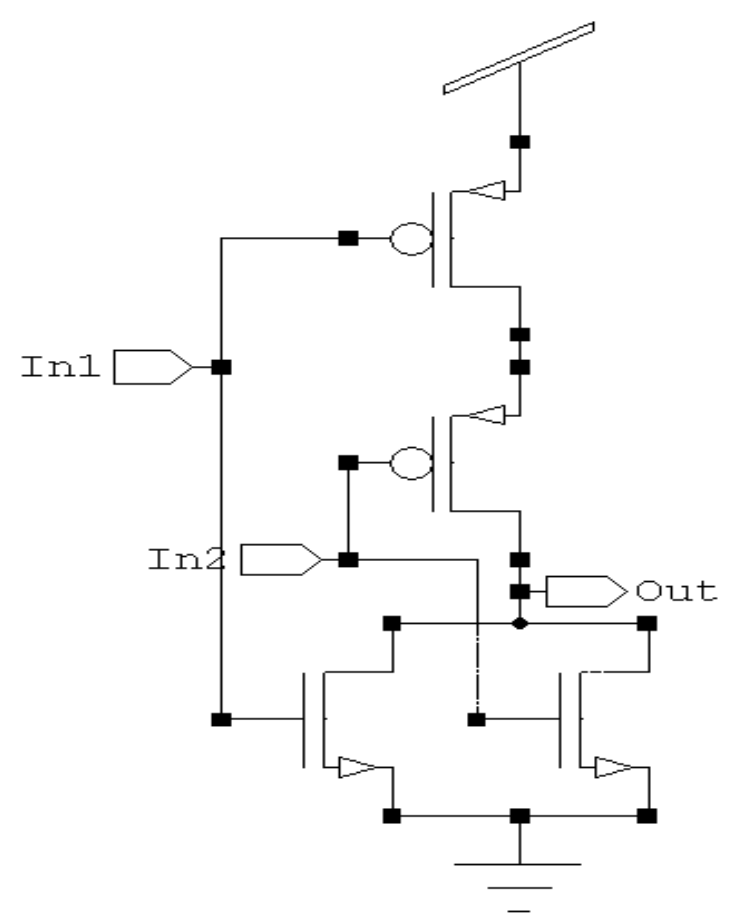

Fig.6 Schematic of NOR Gate 


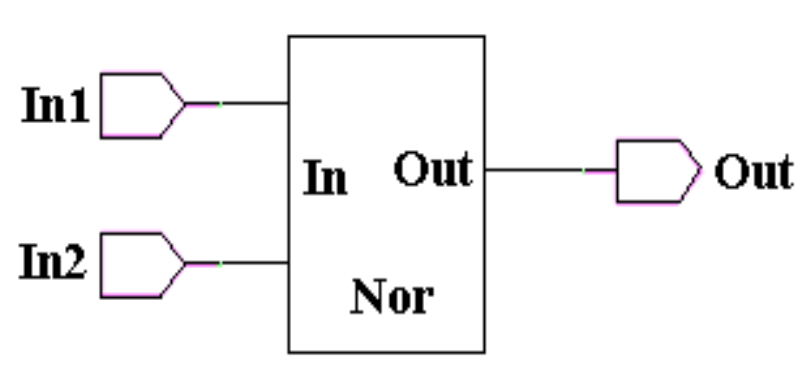

Fig.7 Instance of NOR Gate

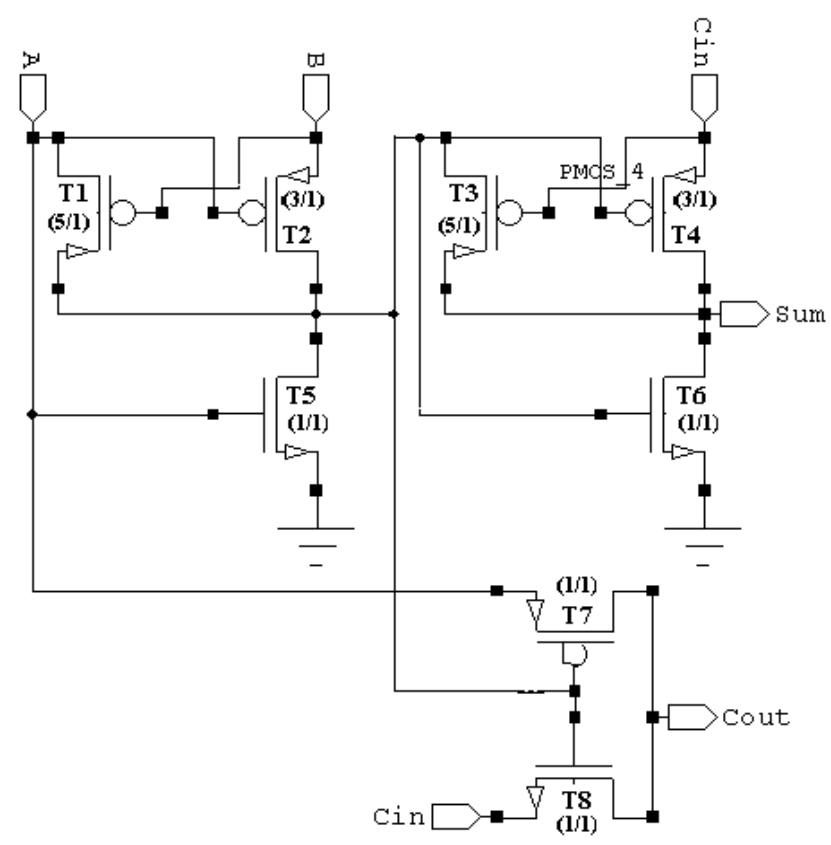

Fig.8 Schematic of existing adder circuit

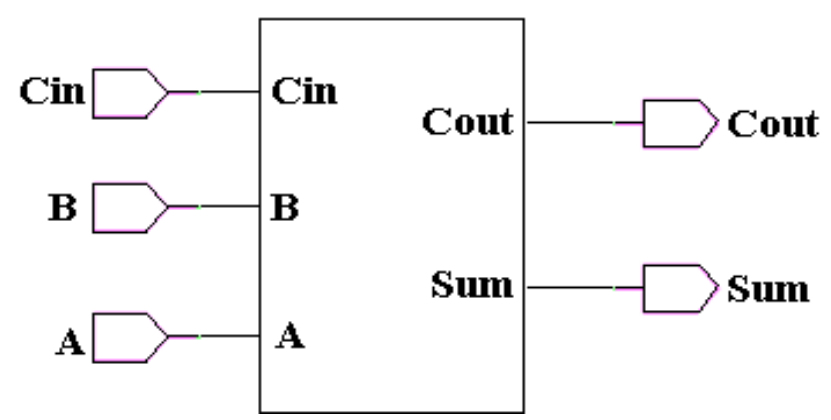

Fig.9 Instance of existing adder

\section{PROPOSED 2X2 ARRAY MULTIPLIER DESIGN}

Fig.10 shows the block diagram of proposed $2 \times 2$ array multiplexer. This diagram contains 8 instances of CMOS inverter, 4 instances of NOR Gate and 2 instances of DCVSL based one bit full adder cell. The instance and schematic of DCVSL based one bit full adder cell is shown in Fig.11 and Fig.12 respectively.[6]-[10]

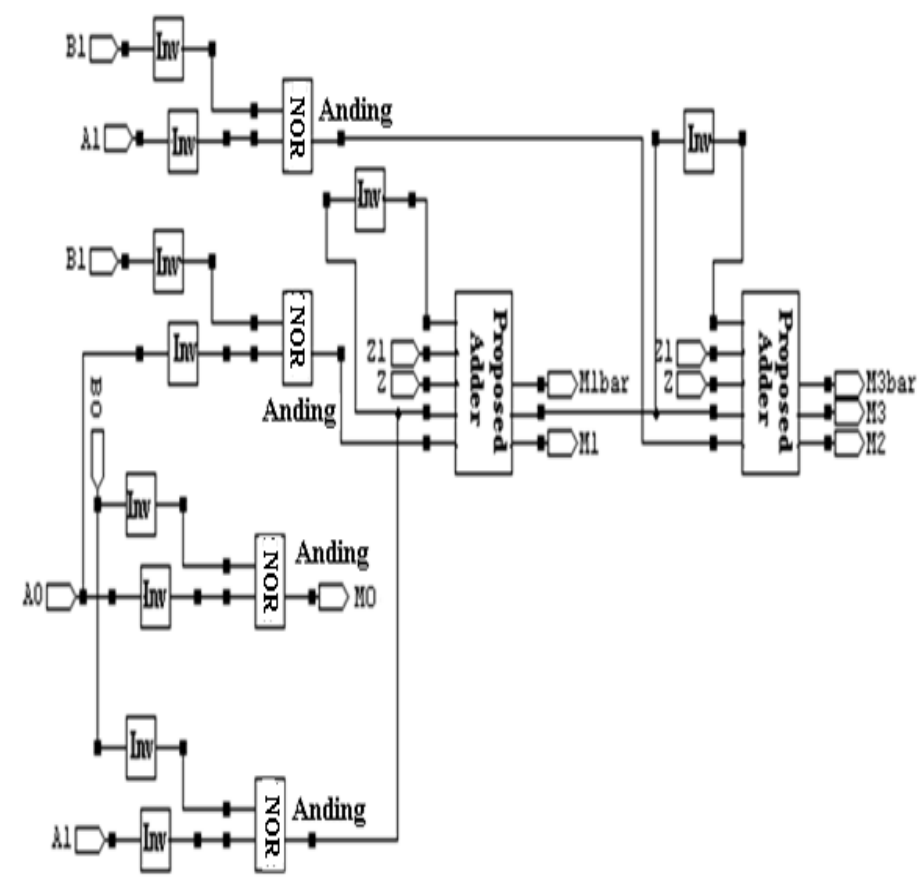

Fig.10 Block diagram of proposed 2x2 multiplier

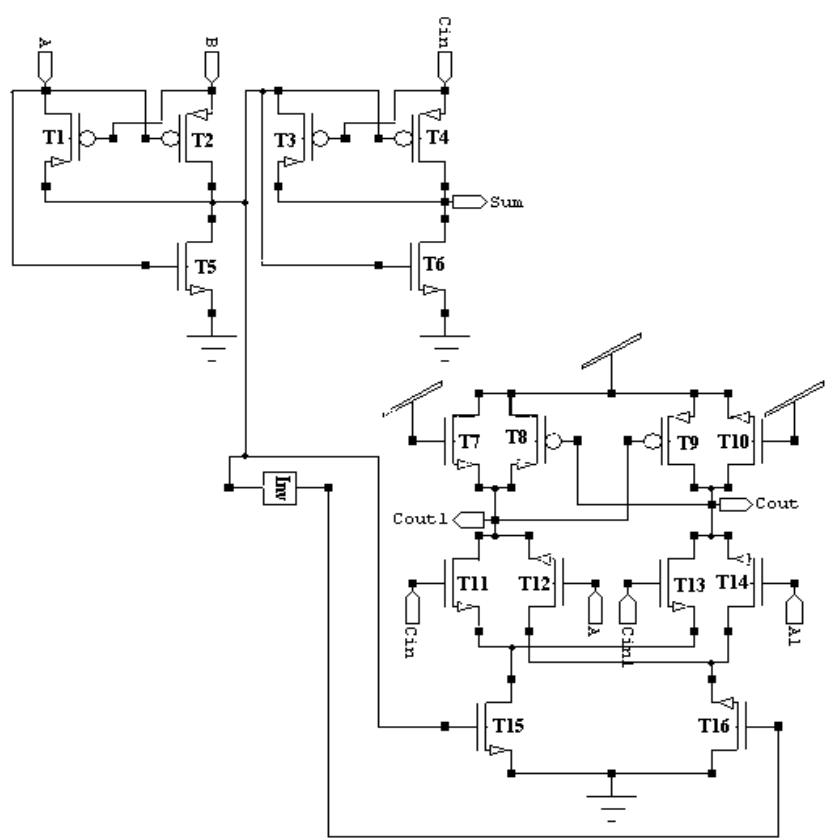

Fig.11 Schematic of adder circuit using DCVSL based 2:1 multiplexer

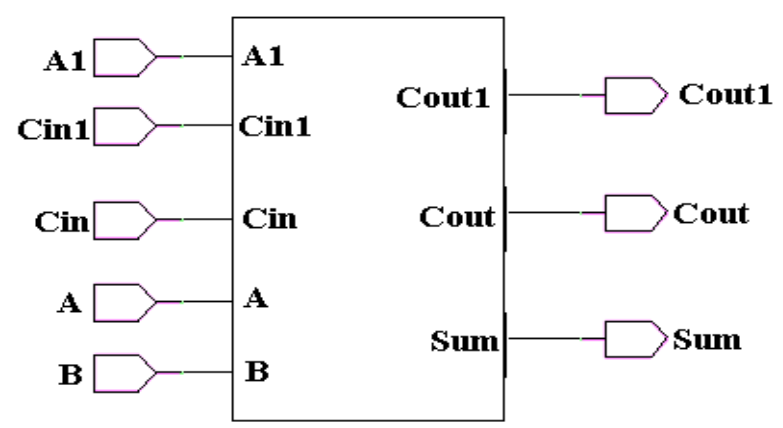

Fig. 12 Instance of DCVSL based adder 


\section{SIMULATIONS AND COMPARISON}

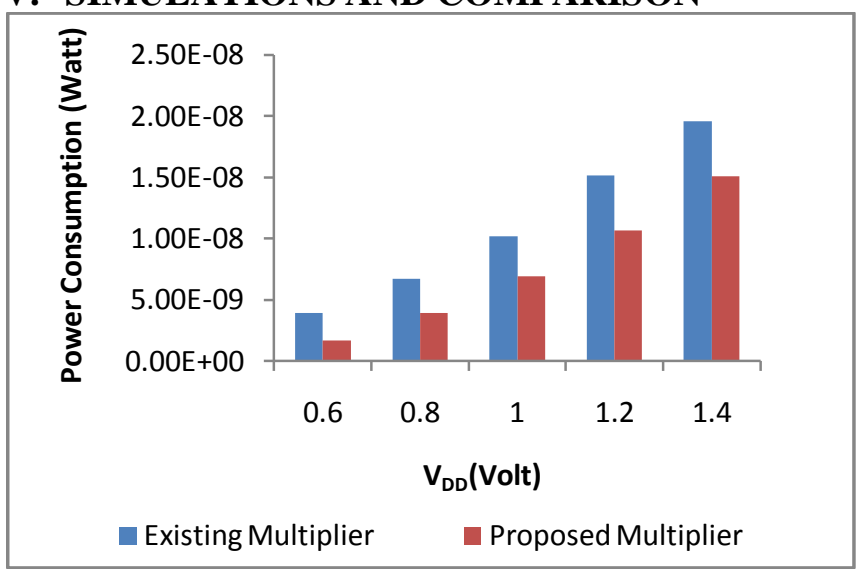

Fig.13 Power consumption comparison of existing and proposed multiplier at different supply voltages

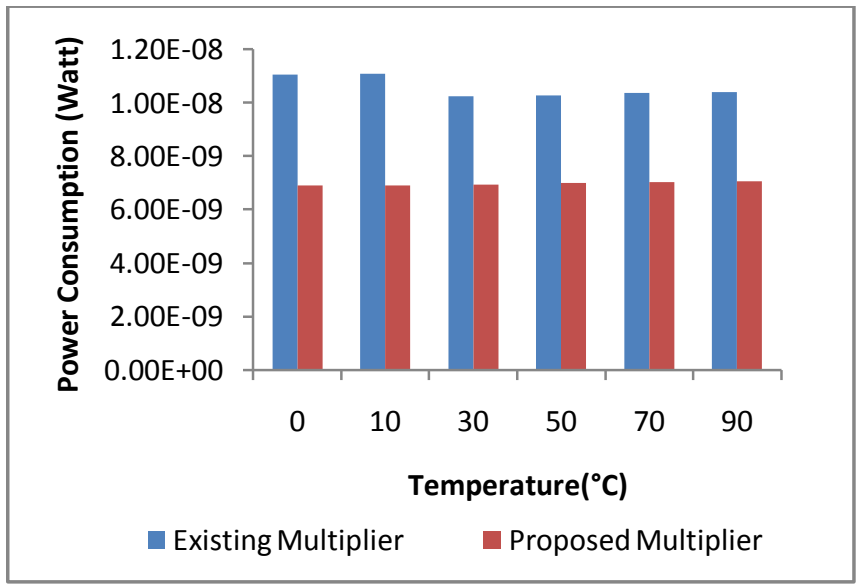

Fig.14 Power consumption comparison of existing and proposed multiplier at different Temperatures

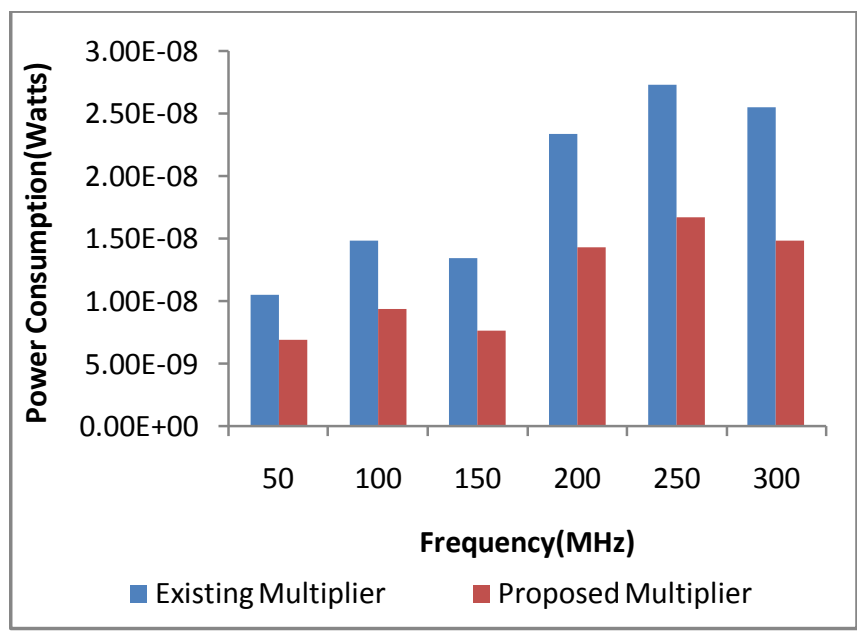

Fig.15 Power consumption comparison of existing and proposed multiplier at different frequencies
The pre-layout simulations are done at $90 \mathrm{~nm}$ technology. Graphs shown in Fig.13-Fig.15 depicts that the proposed $2 \times 2$ array multiplier is the viable option for efficient design. The graph shown in Fig.13 reveals that the power consumption of proposed $2 \times 2$ array multiplier is remarkably less than the existing $2 \times 2$ array multiplier for the input voltage ranging from $0.6 \mathrm{~V}$ to $1.4 \mathrm{~V}$. Fig. 14 and Fig. 15 show graph between power consumption vs. temperature and power consumption vs. frequency, which shows proposed $2 \times 2$ array multiplier based on proposed 2:1 multiplexer has better performance.

\section{CONCluSion}

The $2 \mathrm{X} 2$ array multiplier based on DCVSL one bit full adder cell gives better performance than the existing $2 \mathrm{X} 2$ array multiplier. The $2 \mathrm{X} 2$ array multiplier based on DCVSL one bit full adder cell has been tested and it shows better performance at different temperature and frequency. It shows less power consumption to achieve high performance. It has been designed using 90nm technology and proved it to be a better option for low power complex system design. The net effect is that $2 \mathrm{X} 2$ array multiplier based on DCVSL one bit full adder cell shows a much better performance compared to $2 \mathrm{X} 2$ array multiplier.

\section{REFERENCES}

[1] John P.Uyemura, "Introduction to VLSI Circuits and Systems", John Wiley \& Sons, Inc.

[2] D. Z. Turker, S. P.Khatri, "A DCVSL Delay Cell for Fast Low Power Frequency Synthesis Applications", IEEE transactions on circuits and systems june 2011, vol. 58, no. 6, pp. 1125-1138.

[3] P. K. Lala and A. Walker, "A Fine Grain Configurable Logic Block for Self-checking FPGAs", VLSI Design 2001, Vol. 12, No. 4, pp. 527536.

[4] Jan M. Rabaey, Digital Integrated Circuits; a design prospective, Upper Saddle River: Prentice-Hall, 1996.

[5] T. Sharma, Prof. B.P.Singh, K.G.Sharma, N. Arora "High Speed Array Multipliers Based on 1-Bit Full Adders", in Int. J. of Recent Trends in Engineering and Technology, Vol. 4, No. 4, pp.26-28, Nov 2010.

[6] Heller, L. G. et al., "Cascode Voltage Switch Logic: A differential CMOS Logic Family", proceedings of 1984 IEEE International Solid-state Circuits Conference, pp. 16-17.

[7] Ila Gupta, Neha Arora, Prof.B.P.Singh, "Simulation and Analysis of 2:1 Multiplexer Circuits at 90nm Technology" in International Journal of Modern Engineering Research, Vol.1, Issue.2, pp-642-647, ISSN: 2249-6645, 2011.

[8] Ila Gupta, Neha Arora, Prof.B.P.Singh, "Analysis of Several 2:1 Multiplexer Circuits at $90 \mathrm{~nm}$ and $45 \mathrm{~nm}$ Technologies" International Journal of Scientific and Research Publications, Vol.2, Issue.2, February 2012, ISSN: 2250-3153.

[9] Ila Gupta, Neha Arora, Prof.B.P.Singh, "Design and Analysis of 2:1 Multiplexer for High Performance Digital Systems" International Journal on 
IOSR Journal of Engineering

Apr. 2012, Vol. 2(4) pp: 923-927

Electronics \& Communication Technology (IJECT) Vol.3, Issue1, Jan- March 2012, pp-183-186, ISSN : 2230-7109 (Online)|ISSN : 2230-9543 (Print).

[10] Ila Gupta, Neha Arora, Prof.B.P.Singh, "Low Power 2:1 Multiplexer Design Using DCVS Logic and Its Application in 1-Bit Full Adder Cell" in International Conference on Electronics and Communication Engineering (ICECE), March 03, 2012, Bhopal, India. 